\title{
RESULTADOS DE UN PROGRAMA DE SEGUIMIENTO DE LAS PRÓTESIS MAMARIAS PIP (POL Y-IMPLANT-PROTHESE)*
}

\author{
Drs. Inmaculada Oller N. ${ }^{1}$, María del Pilar Cansado M. ${ }^{1}$, Jaime Ruiz-Tovar P. ${ }^{1}$, \\ Lorea Zubiaga del T. ${ }^{1}$, Antonio Arroyo S. ${ }^{1}$, Rafael Calpena R. ${ }^{1}$ \\ 1 Hospital General Universitario de Elche, Elche, Alicante. \\ España.
}

\begin{abstract}
Results of a program to monitor the PIP breast prostheses (poly-implant-prothese)

Objective: The rupture of mammary protheses PIP (poly-implant-prothese) caused an alarm, settling in Spain a protocol for the management of patients. As hospital unit of reference for the management, monitoring and treatment of patients carrying PIP breast implants, we propose objective to make a descriptive study of the current situation in our country and compare it to the hitherto described in the literature. Method: We conducted a transversal study of all patients who came during the years 2012 and 2013, to the Unit of Mammary Pathology of the Universitary General Hospital of Elche. Results: With a total of 285 women, we observed a high rate of implant rupture $(50.2 \%)$ asymptomatic most $(84.6 \%)$. Fact that the year 2006 had the highest percentage of breakage and the appearance of siliconomas. Conclusions: Our series confirms rupture rate similar to that described in the literature for PIP breast implants. We observed a higher percentage of breakage from the year 2006, coinciding with the period of manufacture described poorer quality of these implants. Axillary siliconomas were evident in $35.1 \%$ of patients, the only locally axillary symptoms.
\end{abstract}

Key words: Breast prostheses, PIP, break, siliconomas.

\section{Resumen}

Objetivo: La rotura de las prótesis mamarias PIP (poly-implant-prothese) ha supuesto una alarma, estableciéndose en España un protocolo para el manejo y seguimiento de las pacientes. Como unidad hospitalaria de referencia para el manejo, seguimiento y tratamiento de las pacientes portadoras de prótesis mamarias PIP, nos proponemos como objetivo realizar un estudio descriptivo de la situación actual en nuestro medio y compararlo con lo hasta ahora descrito en la literatura. Método: Realizamos un estudio transversal de todas las pacientes que acudieron durante los años 2012 y 2013, a la Consulta de la Unidad de Patología Mamaria del Hospital General Universitario de Elche. Resultados: Con un total de 285 mujeres, observamos la elevada tasa de ruptura de los implantes $(50,2 \%)$ la mayoría asintomáticos $(84,6 \%)$. Siendo el año 2006 el de mayor porcentaje de rotura y la aparición de siliconomas. Conclusiones: Se confirma una tasa de ruptura similar a la descrita en la literatura para los implantes mamarios PIP. Observamos un mayor porcentaje de rotura a partir del año 2006, coincidiendo con el período de fabricación descrito de peor calidad de estos implantes. Se evidenciaron siliconomas axilares en el 35,1\% de las pacientes, siendo la única sintomatología a nivel local axilar.

Palabras clave: Prótesis mamarias, PIP, ruptura, siliconomas.

*Recibido el 19 de mayo de 2014 y aceptado para publicación el 22 de septiembre de 2014.

Los autores no refieren conflictos de interés.

Correspondencia: Dra. Inmaculada Oller Navarro.

inma-oller@hotmail.com 


\section{Introducción}

La rotura de los implantes mamarios PIP (PoliImplant-Prothese) ha constituido una alarma social en los últimos años. En España, en el seno del Ministerio de Sanidad Servicios Sociales e Igualdad (MSSSI) se ha establecido un protocolo para el diagnóstico, manejo y seguimiento de las pacientes portadoras de dichas prótesis ${ }^{1}$.

De acuerdo con las conclusiones de las autoridades sanitarias francesas, para la fabricación de las prótesis PIP se utilizaron fraudulentamente materiales de baja calidad (silicona industrial) diferente de la que se había declarado en los documentos presentados para la conformidad de evaluación (silicona médica) ${ }^{2}$. No obstante, la preocupación por la durabilidad de los implantes mamarios de silicona PIP se lleva expresando varios años antes de la aparición de esta alerta ${ }^{3}$. Se estima que aproximadamente 400.000 implantes PIP se vendieron en todo el mundo 4 .

En comparación con otros implantes mamarios de silicona, los cuales se han investigado durante mucho tiempo, para los implantes PIP los datos epidemiológicos disponibles son limitados ${ }^{3,5}$. Sin embargo, la máxima preocupación se ha centrado ahora en la seguridad del dispositivo, la longevidad y una posible asociación con el desarrollo de neoplasias, sobre todo del linfoma anaplásico de células grandes (LACG), aunque su relación parece ser la misma con todas las marcas de prótesis ${ }^{6}$. No obstante, no existe evidencia suficiente que muestre riesgos sanitarios superiores de las prótesis PIP frente a otras prótesis mamarias para las portadoras de estos implantes ${ }^{7,8}$.

Nuestro grupo de trabajo, analizando los datos recogidos como unidad de referencia para el manejo, seguimiento y tratamiento de las pacientes portadoras de prótesis mamarias PIP, nos proponemos como objetivo realizar un estudio descriptivo de la situación actual en nuestro medio y compararlo con lo hasta ahora descrito en la literatura.

El Servicio Nacional Español de Salud Pública, ha garantizado la asistencia a todas las mujeres que han sido implantadas con prótesis mamarias PIP, incluso aquellas implantadas en centros privados o en países extranjeros. Para ello en cada Comunidad Autónoma se han nombrado varios hospitales como unidades de referencia, que serán el punto de partida para iniciar el protocolo de explantación ${ }^{1}$.

\section{Pacientes y Métodos}

Realizamos un estudio transversal de todas las pacientes que acudieron durante los años 2012 y 2013, a la consulta de la Unidad de Patología Ma- maria del Hospital General Universitario de Elche (Alicante) establecido como uno de los centros de referencia en la Comunidad Valenciana.

\section{Protocolo de actuación}

El Ministerio de Sanidad proporcionó un número de teléfono de cada unidad de referencia, donde se les coordinaron las citas en consulta con los distintos especialistas así como para la realización de pruebas de imagen. A todas las pacientes se les realizó una exploración mamaria y axilar, así como una ecografia mamaria, evaluando la rotura protésica y la presencia de siliconomas axilares. Atendiendo a las indicaciones de las autoridades sanitarias, se recomienda el explante si la prótesis está rota. En el caso de existir rotura protésica la indicación fue la de retirar la prótesis mamaria. Si no existía rotura y la paciente no deseaba el explante, se realizará control ecográfico cada 6 meses. En el caso de que las pacientes desearan realizarse un nuevo implante mamario, se les recomendó que acudieran a la medicina privada para realizar en el mismo acto quirúrgico el implante y el explante. En lo referente a los siliconomas, definidos como una reacción granulomatosa como respuesta inflamatoria de los tejidos ante el gel extravasado 9 , las recomendaciones indican eliminar cualquier resto de silicona.

\section{Variables analizadas}

Las variables estudiadas fueron: edad del paciente, año de implantación, tasa de ruptura del implante (especificando bilateralidad), presencia de siliconomas, decisión de explante, reimplante o seguimiento.

\section{Análisis estadístico}

El estudio estadístico se realizó con el programa informático SPSS 19.0 para Windows. Las variables cuantitativas fueron definidas por media y desviación típica (mediana y rango en variables no gaussianas). Las variables discretas fueron definidas por número de casos y porcentaje. Se utilizó el test de $\chi^{2}$ para evaluar la asociación entre variables discretas, cuantificándose la misma mediante la Odds Ratio. Se consideraron valores de $\mathrm{p}<0,05$ como significativos.

\section{Resultados}

Evaluamos un total de 285 mujeres portadoras de implantes mamarios bilaterales PIP (se implantaron un total de 570 prótesis PIP) que fueron atendidas en la Unidad de Patología Mamaria durante los años 2012 y 2013 (pacientes pertenecientes al área sanitaria de Alicante). Todas las pacientes se realizaron el implante en clínicas privadas y por cuestiones esté- 
ticas. Tenían una edad media de 39,6 \pm 10 años. El volumen protésico implantado medio fue de 321,3 $\pm 65,2 \mathrm{ml}$ (rango 180-620).

Atendiendo al año de implantación encontramos prótesis PIP implantadas desde el año 2000 hasta el 2010 en la muestra analizada, con un mayor número de implantes entre los años 2004-2008. El número de implantes durante cada año se expone en la Figura 1 .

Del total de la muestra analizada, fueron $27 \mathrm{mu}-$ jeres $(9,5 \%)$ las que acudieron a consulta con las prótesis PIP ya retiradas, para que se les estudiase y detectase posibles complicaciones derivadas de estos implantes (ejemplo: siliconomas), no descubriéndose éstos en ninguna de ellas. Las pacientes habían decidido retirarse las prótesis al objetivarse rotura demostrada ecográficamente en alguna de ellas. Estas roturas protésicas también fueron incluidas en nuestra serie.

En cuanto a manifestaciones clínicas, sólo 22 mujeres $(7,7 \%)$ presentaban algún tipo de síntoma, manifestado como dolor mamario en 8 de ellas, mastitis en 2 , pérdida de volumen mamario en 2 pacientes y presencia de ganglios axilares palpables en 10 mujeres. Calculando sobre el número total de prótesis implantadas (570) el porcentaje de prótesis sintomáticas se reduciría a 3,8\%.

\section{Hallazgos ecográficos}

La ecografía mamaria mostró prótesis rotas en 143 pacientes $(50,2 \%)$, siendo en 15 de ellas bilateral, en 74 aparecía únicamente la prótesis derecha rota y en 54 la izquierda. Atendiendo al número total de prótesis rotas respecto al total de prótesis implantadas, el porcentaje total de prótesis rotas fue 27,7\% (158 prótesis rotas de 580 implantadas). Ciento veintiuna $(84,6 \%)$ de las pacientes con prótesis rotas no presentaban sintomatología. En todas las pacientes sintomáticas, la ecografía demostró rotura de la prótesis.
Aparecieron ganglios axilares sugestivos de siliconomas en 100 pacientes, 65 casos únicamente en el lado derecho y 21 únicamente en el izquierdo. En 14 pacientes había siliconomas bilaterales. En todos los casos había evidencia de la prótesis ipsilateral rota. Todas las pacientes sintomáticas presentaban siliconomas axilares, sin embargo, estos eran sintomáticos sólo en el $22 \%$ de los casos. Las pacientes sintomáticas tienen 5,2 veces (IC 95\% (2,1$12,9)$ ) mayor probabilidad de tener un siliconoma $(\mathrm{p}<0,001)$. La mediana de siliconomas axilares era de 1 , aunque en un $15 \%$ de los casos había más de un siliconoma en la misma axila.

\section{Decisión de las pacientes}

Del total de pacientes entrevistadas, 100 mujeres $(35,1 \%)$ expresaron la decisión de sustituir las prótesis por un nuevo implante, debiendo ser éste realizado en la medicina privada. Noventa y tres mujeres $(32,6 \%)$ decidieron únicamente el explante sin sustitución. El resto de las pacientes, 92 (32,3\%) y siempre que la ecografía mostrara que las prótesis no estaban rotas, decidieron seguimiento ecográfico cada 6 meses, siendo realizados en sus centros de referencia al igual que el explante mamario. De todas las pacientes con evidencia de prótesis rota (143), 53 deciden reimplantarse y 90 sólo explante. Sólo se realizó seguimiento a las pacientes que decidieron no explantarse. De todas las pacientes con sintomatología clínica (22), 5 deciden reimplantarse y 17 sólo el explante.

Se realizó exéresis de siliconomas sólo en 6 pacientes (extirpando de 1 a 3 siliconomas por paciente), siendo las principales causas para la decisión de la cirugía la ansiedad de la paciente, siliconomas palpables o sintomáticos.

A partir de 2006, se modificó el contenido de silicona de las prótesis PIP, siendo éste de peor calidad. Evaluando por separado las pacientes intervenidas a partir de 2006 y comparándolo con el

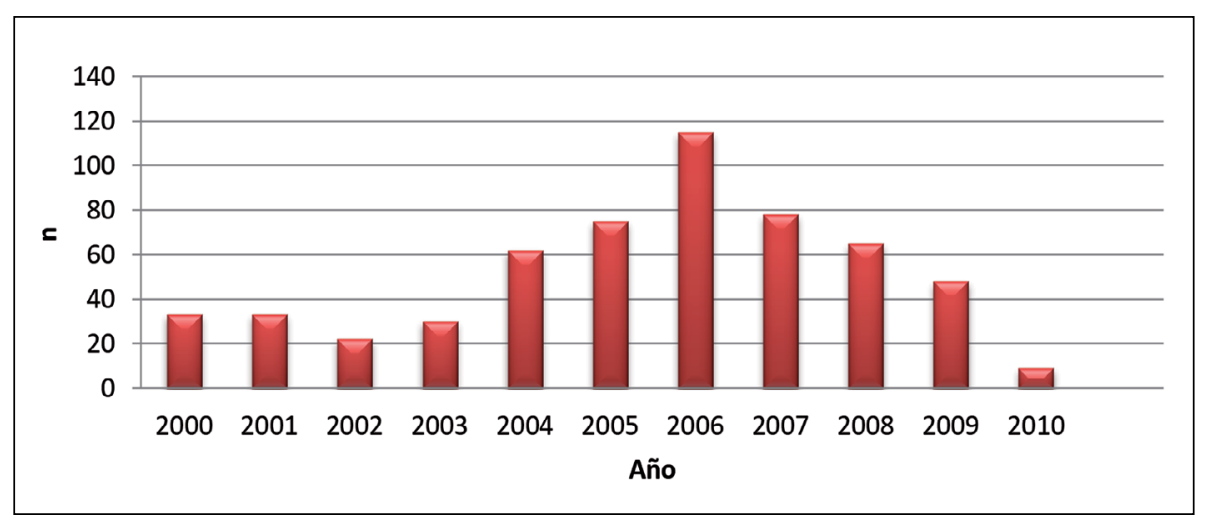

Figura 1. Número de implantes mamarios PIP por años. 


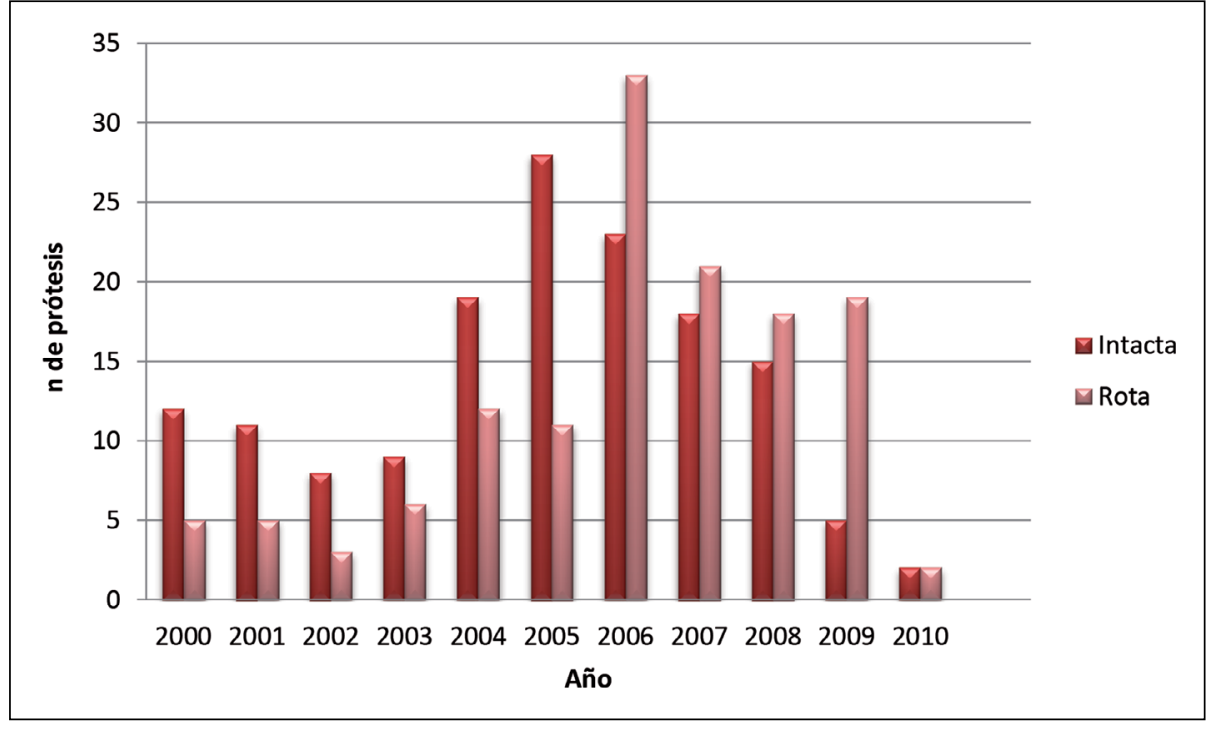

Figura 2. Número de prótesis rotas en función del año de implante. período previo, observamos, independientemente del número de prótesis implantadas, el riesgo de rotura es significativamente superior a partir de 2006 (32\% rotura en pacientes implantadas antes y $59 \%$ rotura a partir de 2006) en los implantes, con una Odds Ratio 1,75 (IC 95\% $(1,1-2,8) \mathrm{p}=0,029)$ (Figura 2). Clínicamente no se encontraron diferencias en sintomatología entre los distintos años de implantación $(\mathrm{p}=0,249)$. Sin embargo, el riesgo de aparición de siliconomas también es significativamente mayor a partir de 2006 (OR 1,8 (IC 95\% (1,1-2,9) p = 0,03).

\section{Discusión}

Los datos disponibles hasta la fecha sobre los implantes mamarios de silicona PIP son limitados y los datos sobre el comportamiento, tasas de fracaso y complicaciones relacionadas con los implantes se basan fundamentalmente en series de $\operatorname{casos}^{4,10}$.

Varios estudios de composición del material implantado, comparando los implantes PIP con el resto de implantes mamarios del mercado, destacan diferencias fundamentalmente en el número de cubiertas de la prótesis y en la composición del gel. Las pruebas realizadas por las autoridades francesas sobre la integridad física de una muestra de los implantes mamarios de silicona PIP indicaron debilidad en la cubierta de las prótesis PIP, no evidenciada en el resto de implantes disponibles en el mercado ${ }^{11}$.

Hasta la fecha las pruebas que se han diseñado para evaluar la citotoxicidad, genotoxicidad e irritación para los geles de silicona médica dan resultados negativos. En el caso de la silicona con- tenida en los implantes PIP, los resultados también fueron negativos para citotoxicidad y genotoxicidad. Sin embargo, pruebas de irritación in vivo fueron positivas $^{12}$. La magnitud dependerá de la cantidad liberada, la duración de la exposición y otras condiciones locales.

En la literatura existe un meta-análisis que demuestra que la rotura de los implantes mamarios en general está directamente relacionada con la duración del implante; $26 \%$ rotura en 3,9 años, $47 \%$ a los 10,3 años, y $69 \%$ a los 17,8 años ${ }^{13}$. Para los implantes mamarios PIP, el último informe de expertos en $2012^{14}$ establece un $31 \%$ de rotura a los 5 años ${ }^{15} y$ aparición de síntomas en el $17 \%$ de los casos con rotura, manifestados fundamentalmente como linfadenopatía patológica, también denominada siliconoma. En nuestra serie se objetivó una tasa de rotura del $27 \%$, datos similares a los descritos en la literatura. Sin embargo, 85 pacientes desarrollaron siliconomas de un total de 158 prótesis rotas $(53,8 \%)$. Esta tasa de siliconomas es muy superior a la descrita en los estudios previos, si bien hay que tener en cuenta, que en nuestra serie sólo el 7\% de los pacientes presentaban síntomas relacionados con los siliconomas y los demás han sido meros hallazgos de la ecografía realizada como screening.

Aunque la manifestación clínica más frecuente de rotura es el siliconoma axilar, también puede manifestarse como seroma, dolor mamario, asimetría, pérdida de consistencia o tumoración en la mama ${ }^{16-18}$. En nuestro grupo sólo se desarrolló clínica loco-regional en 22 pacientes $(15,4 \%)$ con rotura protésica, siendo los síntomas predominantes 
los derivados de los siliconomas axilares, seguido por dolor mamario, pérdida de volumen y un caso de mastitis, confirmando los datos descritos en la literatura.

La rotura del implante del lado derecho mostró un porcentaje superior de rotura respecto a las prótesis contralaterales. La explicación más racional para este hecho parece recaer en un origen traumático. Partiendo de la base de que la mayoría de nuestras pacientes eran diestras, es lógico pensar que la mayoría de traumatismos, esfuerzos y tensiones se realicen con este lado del cuerpo, por lo que las posibilidades de rotura se incrementan ante el mayor estrés traumático.

Algunos autores han descrito la presencia de siliconomas en ausencia de rotura protésica ecográficamente visible, hecho que parece corresponder a que la rotura pueda pasar desapercibida en la prueba de imagen, aunque también se ha comprobado la filtración de silicona a través de la cubierta en ausencia de rotura ${ }^{11}$. No obstante, en nuestras pacientes los siliconomas aparecieron sólo en casos con evidencia de rotura protésica. Los siliconomas aparecen con mayor frecuencia en axila derecha, coincidiendo con el lado con más roturas ecográficamente visualizadas.

Se ha evidenciado que las prótesis implantadas a partir de 2006 contenían una mayor cuantía de silicona industrial y, por tanto, sus características orgánicas se veían alteradas. En nuestras pacientes hemos observado una mayor tasa de rotura en las prótesis implantadas durante ese período, así como un mayor número de siliconomas detectados. Al igual que aparecen casos descritos de siliconomas en otras localizaciones (hepáticos, cutáneos o intrapulmonares) ${ }^{19}$, en la muestra analizada observamos la presencia de siliconomas axilares y se identificaron adenopatías patológicas en otras localizaciones como la región cervical y supraclavicular. Las últimas recomendaciones establecen extirpar aquellos siliconomas sintomáticos o que causen intranquilidad a las pacientes ${ }^{14}$. En nuestro grupo de trabajo realizamos el tratamiento de los siliconomas, atendiendo a estas recomendaciones. Por ello, solamente se extirparon en 6 pacientes (siendo el número entre 1 y 3 siliconomas por paciente).

Las autoridades sanitarias españolas recomiendan el explante de toda prótesis PIP sólo si presenta ruptura. Sin embargo, el Sistema Nacional de Salud sólo financia el explante, pero no un reimplante de nuevas prótesis, dado que éstas fueron implantadas por motivos estéticos. Por cuestiones meramente económicas, un $32,6 \%$ de las mujeres decidieron sólo explantarse, mientras que un $32,3 \%$, todas ellas sin evidencia de rotura, decidió seguimiento ecográfico semestralmente.

\section{Conclusión}

En la muestra analizada se confirma una tasa de ruptura similar a la descrita en la literatura para los implantes mamarios PIP. Nuestros resultados muestran unas prótesis mamarias de baja calidad con un elevado porcentaje de ruptura. Observamos un mayor porcentaje de rotura a partir del año 2006, coincidiendo con el período de fabricación descrito de peor calidad de estos implantes. Se evidenciaron siliconomas axilares en el $35,1 \%$ de las pacientes, siendo la única sintomatología a nivel local axilar, aún habiendo encontrado restos de silicona en otras localizaciones.

\section{Referencias}

1. www.msc.es. http://www.aemps.gob.es/productosSanitarios/prodSanitarios/infor_PIP/.

2. Gamper TJ, Khoury H, Gottlieb W, Morgan RF. Silicone gel implants in breast augmentation and reconstruction. Ann Plast Surg. 2007;59:581-90.

3. Maijers MC, Niessen FB. Prevalence of rupture in poly implant Prostheses silicone breast implants, recalled from the European market in 2010. Plast Surg Reconstr. 2012;129:1372-8.

4. SCENIHR (scientific committee on emerging and newly identified health risks), European commission, press release, medical devices: European commission asks for further scientific study and draws first lessons from the recent fraud on breast implants, Feb 2012. http://www. eisnersafety.com/2-feb-12-ec-asks-for-further-scientificstudy-draws-1 st-lessons-from-recent-fraud-on-breastimplants/

5. Spear SL, Murphy DK, Slicton A, Walker PS. Inamed silicone breast implant core study results at 6 years. Plast Reconstr Surg. 2007;120:8S-16S.

6. Brody G, Deapen D, Gill P. T-cell non Hodgkin's anaplastic lymphoma associated with one style of breast implants. 89th Annual Meeting, American Association of Plastic Surgeons, San Antonio, TX. 2010.

7. Farace F, Bulla A, Marongiu F, Campus GV, Tanda F, Lissia A, et al. Anaplastic Large Cell Lymphoma of the Breast Arising Around Mammary Implant Capsule: An Italian Report. Aesthetic Plast Surg. 2013;37:567-71.

8. Kim B, Roth C, Young VL, Chung KC, Van Busum K, Schnyer C, et al. Anaplastic large cell lymphoma and breast implants: results from a structured expert consultation process. Plast Reconstr Surg. 2011;128:629-39.

9. Vaamonde R, Cabrera JM, Martín Vaamonde-RJ, Jimena I, Marcos Martín J. Silicone granulomatous lymphadenopathy and siliconomas of the breast. Histol Histopathol. 1997;12:1003-11.

10. Brandon HJ, Young VL, Watson ME, Wolf CJ, Jerina KL. Protocol for retrieval and analysis of breast im- 
plants. J Long-Term Effects Med Implants 2003;13:4961.

11. Reyal F, Feron JG, Leman Detour S, Pourcelot AG, Valentin M, Philippe AC, et al. The impact of poly implant prothèse fraud on breast cancer patients: a report by the Institut Curie. Plast Reconstr Surg. 2013;131:690-5.

12. Brandon HJ, Jerina KL, Wolf CJ, Young VL. In vivo aging characteristics of silicone gel breast implants compared to lot-matched controls. Plast Reconstr Surg. 2002;109:1927-33.

13. Marotta JS, Goldberg EP, Habal MB, Amery DP, Martin PJ, Urbaniak DJ, et al. Silicone gel breast implant failure: evaluation of properties of shells and gels for explanted prostheses and meta-analysis of literature rupture data. Ann Plast Surg. 2002;49:227-42.

14. Poly implant prothese (Pip) breast implants: final report of the expert group, Sir Bruce Keogh, NHS Medical Director, June 2012. http://www.nhs.uk/conditions/ breast-implants/documents/.

15. Berry M, Stanek JJ. The PIP mammary prosthesis: a product recall study. J Plast Reconstr Aesthet Surg. 2012;65:697-704.

16. Berry MG, Stanek JJ. Rupture of a silicone gel mammary prosthesis and amyloidosis: a case report. Aesthetic Plast Surg. 2002;26:383-7.

17. Dieterich M, Stubert J, Stachs A, Radke A, Reimer T, Gerber B. Ruptured poly-implant protheses breast implant after aesthetic breast augmentation: diagnosis, case management, and histologic evaluation. Aesthetic Plast Surg. 2013;37:91-4.

18. Zedníková I, Třešková I, Schmiedhuber P, Hes O. Complications of breast augmentation-a case report. Rozhl Chir. 2012;91:435-7.

19. Dragu A, Theegarten D, Bach AD, Polykandriotis E, Arkudas A, Kneser U, et al. Intrapulmonary and cutaneous siliconomas after silent silicone breast implant failure. Breast J. 2009;15:496-9. 